\title{
Linking Molecular Events to Cellular Responses at Low Dose Exposures
}

(Project Number: 69941)

\section{Principal Investigator}

Thomas J. Weber

Pacific Northwest National Laboratory

P.O. Box 999, MSIN P7-56

Richland, WA 99352

(509) 376-2318 (phone)

Thomas.Weber@exchange.pnl.gov

\section{Co-Investigators}

Nancy H. Colburn

Gene Regulation Section

National Cancer Institute

Bldg. 560, Rm. 21-89

Frederick, MD 21702-1201

(301) 846-1342 (phone)

Colburn@ncifcrf.gov

Michael K. Bowman

Pacific Northwest National Laboratory

P.O. Box 999, MSIN K8-98

Richland, WA 99352

(509) 376-3299 (phone)

Michael.Bowman@exchange.pnl.gov 


\section{Research Objective}

The studies proposed in this project are to define thresholds in cell signaling pathways that are required for cellular transformation and may be targeted by low-dose radiation. Defining thresholds in transformation-related signal transduction pathways that are sensitive to low-dose radiation would be an important advancement in risk assessment and could be used to demonstrate nonlinear relationships between low-dose radiation and cancer.

\section{Research Progress}

This report summarizes work after five months of a three-year project. Efforts are under way to develop the molecular tools required to investigate thresholds in transformation-related signal transduction pathways. Dose response for a polypeptide tumor promoter (epidermal growth factor [EGF]) has been conducted in a 12-O-tetradecanoyl phorbol-13-acetate (TPA) responsive element (TRE)-luciferase reporter cell line to establish the linear range of the TRE-reporter assay for extension to low-dose radiation studies. Exposure conditions for examining dose-rate effects on the transformation response to EGF have been investigated and the exposure system modified to minimize priming effects due to sham exposure.

New stable transfectant JB6 reporter cell lines have been generated. These are to serve as readouts for measuring induced Erk activation or activation of transcription factors AP-1 or NF- $\kappa \mathrm{B}$. Thus far, 4X AP-1-luciferase/JB6 Cl $41 \mathrm{P}+$ cells and 5X NF- $\kappa \mathrm{B}-$ luciferase $\mathrm{Cl} 41$ reporter cells have been generated and drug selected. Multiple clones are about to be screened for low basal activity and greater than two-fold inducibility by TPA. Four reporter clones will be chosen for each transcription factor.

Another readout for activation of Erk is activation of the fusion substrate Gal4-Elk. We have experimental evidence that inhibition of the Erk kinase MEK by U01267 inhibits Erk-dependent Gal4-Elk activation in a dose-dependent fashion. We expect to obtain parallel measurement of phosphoErk levels for each concentration of MEK inhibitor, thus permitting a direct correlation of Elk activation level with concentration of activated (phosphorylated) Erk. Note that Gal4-Fra-1 activation is also dependent on Erk activity. This exciting discovery is the subject of a manuscript soon to be submitted. Transient transfection of SRE-luciferase also demonstrates quantitative dependency on activated Erk levels. Finally AP-1-luciferase reporter activation (transient transfection) is concentration dependently regulated by activated Erk levels. Measurements of superoxide dismutase activity have been optimized using an enzyme-linked immunosorbent assay (ELISA).

\section{Planned Activities}

The construction of a tetracycline-inducible dominant negative Erk construct is under way. We have generated JB6 P+ cells that harbor the tetracycline regulator (G418 selection). Once 
the TRE-DNErk is available it will be transfected into the tet regulator cells (hygromycin selection). These inducible DNErk cells will allow us to regulate the cellular concentration of activated Erk precisely.

Superoxide dismutase activity will be determined in vivo and in vitro following exposure to low-dose gamma radiation. Changes in SOD activity will be confirmed at the protein level by Western blot for specific SOD activities.

Yet to be generated: SRE-luciferase/P+ cells. These will provide quantitative readout for Erk activation. Following full optimization of the radiation exposure system, the effect of dose and dose-rate on transformation by EGF and TPA will be determined.

\section{Information Access}

As mentioned above, the work describing the Erk dependent Gal4-Elk activation is being prepared for publication. 